\title{
1 Particle foraging strategies promote microbial diversity in marine environments
}

2 Authors: Ali Ebrahimi ${ }^{1 \dagger}$, Akshit Goyal $^{2 \dagger}$, Otto X. Cordero ${ }^{1 *}$

3

4 Authors affiliation:

$5 \quad{ }^{1}$ Ralph M. Parsons Laboratory for Environmental Science and Engineering, Department of Civil

6 and Environmental Engineering, Massachusetts Institute of Technology, Cambridge, MA 02139

7 USA

$8{ }^{2}$ Physics of Living Systems, Department of Physics, Massachusetts Institute of Technology,

9 Cambridge, MA 02139, USA

10 * Correspondence to: ottox@mit.edu

$11+$ Equal contribution.

Abstract:

Microbial foraging in patchy environments, where resources are fragmented into particles or pockets embedded in a large matrix, plays a key role in natural environments. In the oceans and freshwater systems, particle-associated bacteria can interact with particle surfaces in different ways: some colonize only during short transients, while others form long-lived, stable colonies. We do not yet understand the ecological mechanisms by which both short-term and long-term colonizers can coexist. Here, we address this problem with a mathematical model that explains how marine populations with different detachment rates from particles can stably coexist. In our model, populations grow only while on particles, but also face the increased risk of mortality by predation and sinking. Key to coexistence is the idea that detachment from particles modulates both net growth and mortality, but in opposite directions, creating a trade-off between them. While slow-detaching populations show the highest growth return (i.e., produce more net offspring), they are more susceptible to suffer higher rates of mortality than fast-detaching populations. Surprisingly, fluctuating environments, manifesting as blooms of particles (favoring growth) and predators (favoring mortality) significantly expand the likelihood that populations with different detachment rates can coexist. Our study shows how the spatial ecology of microbes in the ocean can lead to a predictable diversification of foraging strategies and the coexistence of multiple taxa on a single growth-limiting resource. 


\section{Introduction}

Microbes in nature are remarkably diverse, with thousands of species coexisting in any few milliliters of seawater or grains of soils ${ }^{1,2}$. This extreme diversity is puzzling since it conflicts with classic ecological predictions. This puzzle has classically been termed "the paradox of the plankton," referring to the discrepancy between the measured diversity of planktons in the ocean, and the diversity expected based on the number of limiting nutrients ${ }^{3-6}$. Decades of work have helped, in part, to provide solutions for this paradox in the context of free-living (i.e., planktonic) microbes in the ocean. Many have suggested new sources of diversity, such as spatiotemporal variability, microbial interactions, and grazing ${ }^{7-9}$. However, in contrast with free-living microbes, the diversity of particle-associated microbes - often an order of magnitude greater than freeliving ones - has been overlooked ${ }^{10-12}$. In contrast with planktonic bacteria, which float freely in the ocean and consume nutrients from dissolved organic matter, particle-associated microbes grow by attaching to and consuming small fragments of particulate organic matter (of the order of micrometers to millimeters). It is thus instructive to ask: what factors contribute to the observed diversity of particle-associated microbes, and how do these factors collectively influence the coexistence of particle-associated microbes?

The dispersal strategies of particle-associated microbes can be effectively condensed into one parameter: the rate at which they detach from particles. This rate, which is the inverse of the average time that microbes spend on a particle, is the key trait distinguishing particle-associated microbial populations from planktonic ones ${ }^{13,14}$. The detachment rates of such particle-associated taxa can be quite variable ${ }^{15,16}$. Bacteria with low detachment rates form biofilms on particles for efficient exploitation of the resources locally, while others with high detachment rates frequently attach and detach across many different particles to access new resources ${ }^{17}$. Therefore, the question of how diversity is maintained in particle-associated bacteria is connected with the question of how bacteria with different dispersal rates can coexist. In this study, we address the latter question. Specifically, we ask how two populations with different dispersal strategies can coexist while competing for the same set of particles. We address this question under a range of conditions relevant for marine microbes.

We hypothesize that dispersal is key to the coexistence of particle-associated microbes and thus 66 might explain their high diversity. The degree of species coexistence on particles depends on the 67 balance between growth and mortality. On particles, net mortality rates can be higher than for 
planktonic cells because of the large congregation of cells on particles, which exposes them to the possibility of a large and sudden local population collapse. The collapse of a particle-attached population can be induced by a variety of mechanisms, including particles sinking below a habitable zone ${ }^{18}$, or predation of whole bacterial colonies by viruses or grazers . For instance, after a lytic phage bursts out of a few cells on a particle, virions can rapidly engulf the entire bacterial population, leading to its local demise ${ }^{11,19,20}$. Such particle-wide mortality may kill more than $30 \%$ of particle associated populations in the ocean ${ }^{21,22}$. The longer a population stays on a particle, the higher the chance it will be wiped out. This trade-off between growth and risk of mortality suggests that there could be an optimal residence time on particles. It is however unclear whether such a trade-off could enable the coexistence of populations with different dispersal strategies and, if so, under what conditions.

Here we study this trade-off using mathematical models and stochastic simulations. These models reveal that the trade-off between growth and survival against predation can indeed lead to the stable coexistence of particle-associated microbial populations with different dispersal strategies (in our work, detachment rates). We also study how environmental parameters, such as the supply rate of new particles, determine the dominant dispersal strategy and the range of stable coexistence. Our results show that in bloom conditions, when the particle supply is high, fast dispersers that rapidly hop between particles are favored. In contrast, under oligotrophic conditions, when particles are rare, rarely detaching bacteria have a competitive advantage. Overall, our work shows that differences in dispersal strategies alone can enable the coexistence of particle-associated marine bacteria, in part explaining their impressive natural diversity.

\section{Results}

Overview of the model: To understand how differences in dispersal strategies affect bacterial coexistence, we developed a mathematical model that describes the population dynamics of bacteria colonizing a bath of particles with a chosen dispersal strategy. More specifically, in our model, bacterial cells attach to particles from a free-living population in the bulk of the bath; they then grow and reproduce while attached. Detachment is stochastic with a fixed rate. After detachment, cells re-enter the free-living population and repeat the process. During the time spent attached to particles, all bacteria on a particle may die with a fixed probability per unit time, corresponding to their particle-wide mortality rate (Figure 1A). Another important feature of the model is density-dependent growth, which means that per capita growth rates decrease with 
increasing population size. For this, we use the classic logistic growth equation, which contains a simple linear density dependence (Figure 1B; Methods). Free-living subpopulations cannot grow, but die at a fixed mortality rate due to starvation. The probability of a bacterium encountering particles controls bacterial attachment, which we calculate using random walk theory as the hitting probability of two objects with defined sizes ${ }^{23,24}$ (see Methods for details). We assume that the detachment rate is an intrinsic property of a bacterial population and comprises its dispersal strategy independent of the abiotic environment. In our simulations, it is the only trait that varies between different bacterial populations. Growing evidence has shown that bacterial detachment rates differ significantly across marine bacterial communities from solely planktonic cells to biofilm-forming cells on particles ${ }^{17,25}$. Using this mathematical model, we asked how variation in detachment rate affects bacterial growth dynamics and the ability of multiple subpopulations to coexist on particles. For this, we simulated bacterial population dynamics on a bath of several particles and measured each population's relative abundance at a steady state (example in Figure $1 \mathrm{C})$.

Bacterial mortality determines optimal foraging strategies: Our model simulates growth, competition and dispersal in a patchy landscape, similar to classical models of resource foraging, with the additional element of mortality, both within and outside patches (i.e., particles). We hypothesized that the inclusion of mortality could play an important role in affecting the success of a dispersal strategy (i.e., detachment rate), since it would alter the cost of staying on a particle. To investigate how mortality affects dispersal strategies, we studied its effect on the optimal strategy, which forms the focus of many classical models of foraging. According to optimal foraging theory (OFT), the optimal time spent on a particle is one that balances the time spent without food while searching for a new patch, with the diminishing returns from staying on a continuously depleting patch ${ }^{26,27}$. In our model, particles are analogous to resource patches, and the detachment rate is simply the inverse of the time spent on a particle (residence time). 
(A)

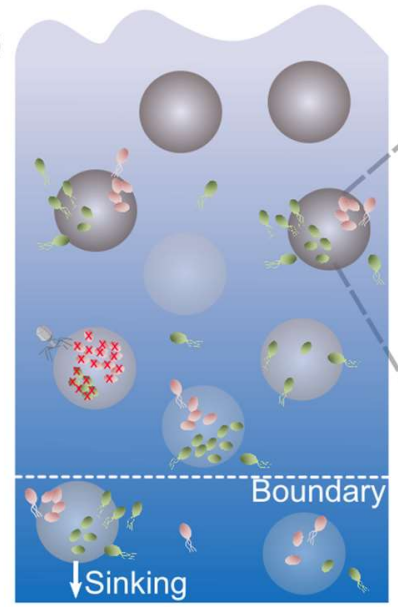

(B)

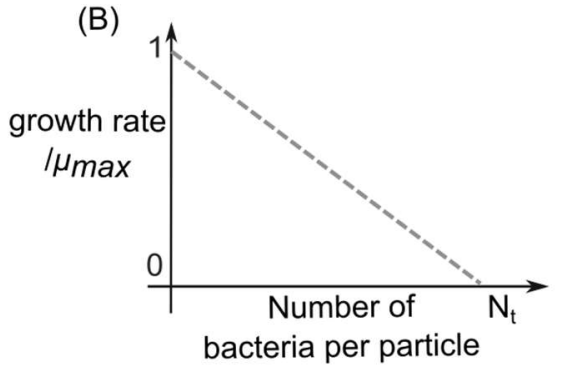

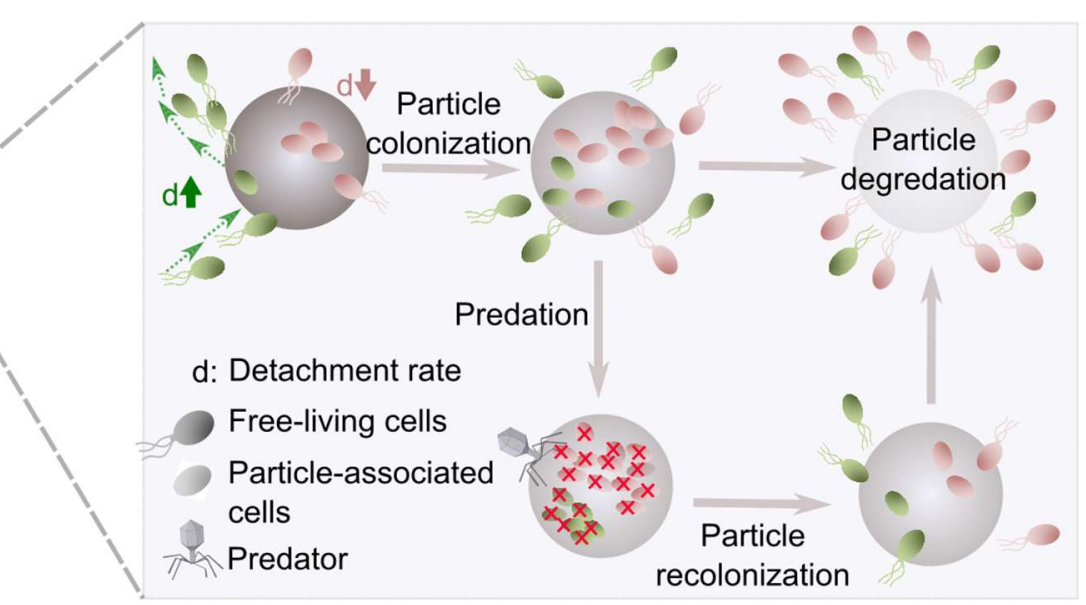

(C)

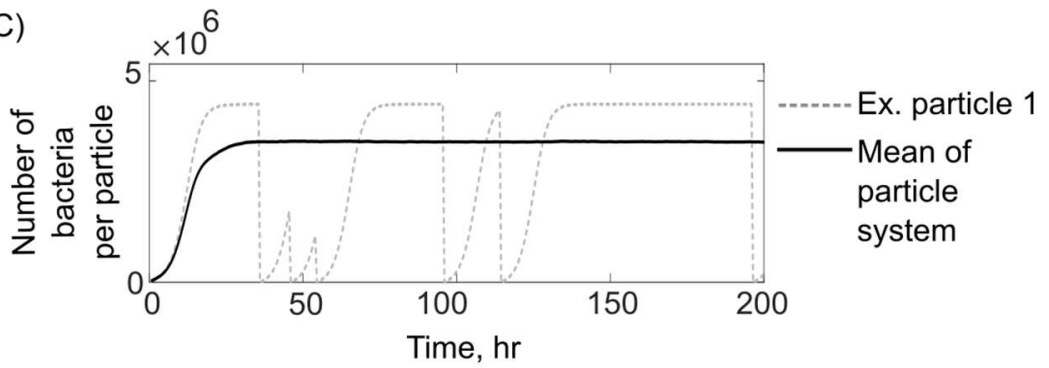

Figure 1: Schematic representation of the mathematical model simulating slow and fast

The model assumes that the predation on a particle or sinking out of system's boundaries kills it's all associated populations. After infection, the non-colonized particle is then recolonized by free-living populations. As resources on a particle are consumed, its associated populations are dispersed and are added to the free-living populations. In this case, the old particle is replaced by a new un-colonized particle in the system. (B) The growth kinetics on a single particle is assumed to be density-dependent and decreases linearly as a function of the number of cells colonizing the particle. $N_{t}$ represents the carrying capacity of the particle. C) The dynamics of particle-associated cells and their corresponding growth rates are shown for a system with 1000 particles. The mean values over many particles and an example of dynamics on a single particle are illustrated.

As expected, OFT predicts the optimal detachment rate given a distribution of resources and search times, but only in the absence of mortality (Figure 2A). To test if our model agrees with 
the predictions of OFT, we calculated the optimal detachment rate $\left(d_{\text {opt }}\right)$ using simulations of our model in the absence of mortality and compared it with OFT predictions (Methods). We found that the optimal detachment rate, which outcompetes all other detachment rates, was consistent with OFT predictions across a wide range of particle numbers in our system (Figure 2A). Strikingly, in the presence of mortality, the optimal detachment rate $\left(d_{o p t}\right)$ changed significantly, either increasing or decreasing depending on the type of mortality. When mortality was particle-wide, the optimal detachment rate was much higher than predicted by OFT, often resulting in residence times that were many days shorter than the OFT prediction (Figure 2A). This is because it is more beneficial to detach faster when there is a higher risk of particle-wide extinction. In contrast, when mortality was only present in free-living populations (affecting individuals, not particles, at a constant per capita rate), the optimal detachment rate was much lower than predicted by OFT (Figure 2A). These results expand on our knowledge of OFT, and explain that the source and strength of mortality - on individuals or on whole particles - can differently impact the optimal detachment rate.

(A)

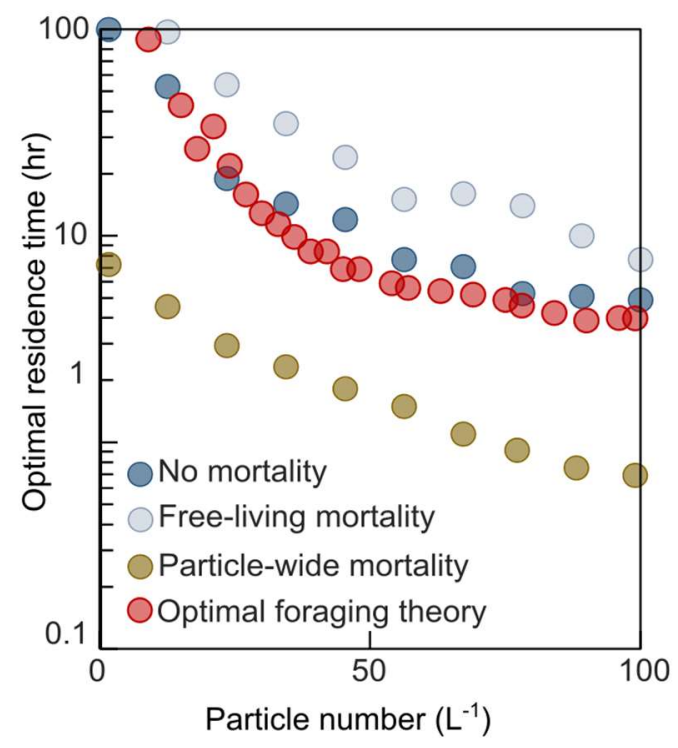

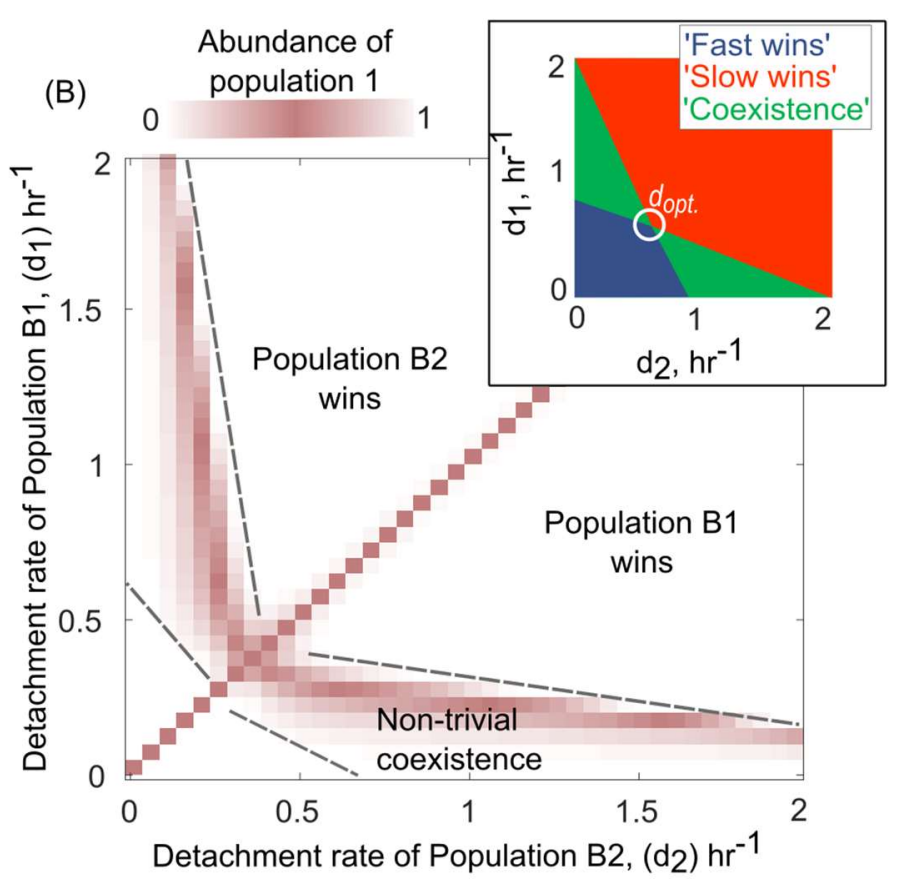

Figure 2. Variation in bacterial detachment strategies allow coexistence in the particle system. (A) Optimal residence time predicted by the population-based model and optimal foraging theory (Methods). Three scenarios with various particle-wide mortality $\left(m_{p}\right)$ and mortality on free living populations $\left(m_{F}\right)$ are simulated with the following rates: (i) particle-wide mortality $\left(m_{p}=0.05 h r^{-1}, m_{F}=0.02 h r^{-1}\right)$, (ii) free living mortality $\left(m_{p}=0 h r^{-1}, m_{F}=0.05 h r^{-1}\right)$ (iii) no mortality $\left(m_{p}=0 \mathrm{hr}^{-1}, m_{F}=0 \mathrm{hr}^{-1}\right.$. To calculate optimal residence time based on optimal foraging 
theory (OFT), we used our model and tracked individual cells attaching to a particle. The timeaveraged uptake rate of the attached cell and its instantaneous uptake rate were calculated.

178 The residence time with similar instantaneous and time-averaged uptake rates is assumed to be optimal residence time based on OFT (see method for details). In our population-based model, the optimal residence time is assumed to be a residence time that maximizes the growth return from the particles. (B) The relative abundance of population one is shown for competition experiments of two populations with different detachment rates. The relative abundance is measured at the equilibrium, where no changes in the sizes of both populations are observed.

The area with white color represents the conditions where either one of the populations is extinct. The mortally on particles is assumed $0.02 \mathrm{hr}^{-1}$. (inset) Phase diagram of the coexistence as a function of detachment rates for two competing populations. $d_{o p t .}$ represents the optimal detachment rate that the coexistence range nears zero. (B) The attachment rates are kept constant at $0.0005 \mathrm{hr}^{-1}$. The number of particles is assumed to be $60 \mathrm{~L}^{-1}$. The carrying capacity of the particle is assumed to be $5 e 10^{6}$. Simulations are performed using our population-based mathematical model.

\section{A trade-off between growth and mortality enables the coexistence of dispersal strategies:} Having observed that mortality can greatly affect the success of a dispersal strategy, we next sought to understand whether it could enable the coexistence of bacterial populations with different strategies (detachment rates). Simulations where we competed a pair of bacterial populations with different detachment rates revealed that differences in detachment rates alone are sufficient to enable coexistence on particles (Figure 2B). We assessed coexistence by measuring the relative abundances of populations at equilibrium (Figure S1). Interestingly, such a non-trivial coexistence only emerged in the presence of particle-wide mortality. In the absence of mortality on particles, we only observed trivial coexistence (coexisting populations had identical detachment rates, and for the purposes of the model, were one and the same; Figure S2). These results suggested that the presence of particle-wide mortality, where the entire population on a particle suffers rapid death, was crucial for populations with different dispersal strategies to coexist.

To investigate the underlying mechanisms that may give rise to the coexistence of populations with different detachment rates, we quantified the growth return of particle-associated populations

208 as well as their survival rate on particles (Figure 3A-3B). We calculated the average growth return 209 based on the average number of offspring produced per capita during one single attachment- 
detachment event. The survival rate on particles was obtained by subtracting the mortality rate per capita from the offspring production rate per capita (Figure 3B; see Methods). The results revealed that a trade-off between bacterial growth return and survival rate emerged on particles,

213 supporting the coexistence of populations with different detachment rates (Figure 3C-D).

214 Populations that detach slowly from particles have higher growth returns but are also more 215 susceptible to particle-associated mortality. In contrast, populations with low residence time on 216 particles (high detachment rate) have low growth returns but they are less likely to die by predation 217 or sink beyond the habitable zone. We next investigated whether such a trade-off was necessary 218 to enable coexistence in our model.

We developed a coarse-grained model to address the conditions under which we might observe coexistence between populations whose only intrinsic difference was their detachment rates in our system. Our simple model expands on classical literature which describes coexistence among various dispersal strategies in spatially structured habitats ${ }^{28-31}$. We simplified many details in favor of analytical tractability. Chiefly, we assumed that the growth dynamics on each particle were much faster than the dispersal dynamics across particles. This allowed us to replace detailed growth dynamics on single particles with a single number quantifying the bacterial population, $N$, after growth on each particle. In the model, we considered two particle associated populations that competed for a shared pool of particles. To keep track of populations, we quantified the number of particles they had successfully colonized as $B_{1}$ and $B_{2}$, respectively. Individuals from both populations could detach from particles they had already colonized and migrate to a number $E$ of yet-unoccupied particles, with a rate proportional to their detachment rates, $d_{1}$ and $d_{2}$, respectively. Once migrated, individuals rapidly grew on unoccupied particles to their fixed per particle growth returns, $N_{1}$ and $N_{2}$. To model particle-wide mortality, we assumed a fixed per particle mortality rate, $m_{p}$. The population dynamics for the system of particles could therefore be written as follows:

239 At equilibrium $\left(\frac{\partial B_{i}}{\partial t}=0 \forall i\right)$, either population can survive in the system if and only if its net 240 colonization and mortality rates are equal $\left(N_{i} d_{i} E \approx m_{p}\right)$. Consequently, the product of the growth 241 return per particle and the detachment rate of either population should be equal $\left(N_{1} d_{1} \approx N_{2} d_{2}\right)$. 
242 By simplifying Eq. 1 at equilibrium, this model predicts that for two competing populations to

243 coexist, their growth returns and detachment rates on particles must follow the relation:

$$
\frac{N_{1}}{N_{2}} \propto \frac{d_{2}}{d_{1}} .
$$

247 This relationship shows that coexistence demands a trade-off between the growth return $(N)$ of a 248 bacterial population, and its detachment rate $(d)$, i.e., the inverse of an individual's residence time 249 on a particle. In other words, coexistence only emerges when the growth returns increases with 250 the residence time on the particle $\left(\frac{N_{1}}{N_{2}} \sim \frac{T_{1}}{T_{2}}\right)$. In agreement with this, simulations from our detailed 251 model revealed that coexistence between two populations with different detachment rates only 252 occurred in conditions where the two populations obeyed such a relationship, or trade-off (Figure $2533 \mathrm{C})$.

255 While the trade-off allows coexistence, it does not hold across all parameter values, and does not 256 allow any pair of detachment rates to coexist. In particular, no detachment rate can coexist with 257 the optimal detachment rate, thus rendering coexistence between any other set of detachment 258 rates susceptible to invasion by this optimal strategy. Therefore, if detachment rates were allowed 259 to evolve, only one population would survive in the long run - the one with the optimal detachment 260 rate (Figure S3). Motivated by this observation, we next asked whether environmental fluctuations 261 would render coexistence evolutionarily stable, or whether they would further destabilize the 262 coexistence of populations with non-optimal dispersal strategies. 
(A)

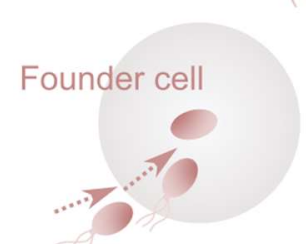

Attachment event

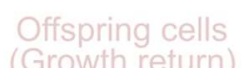

(Growth return)

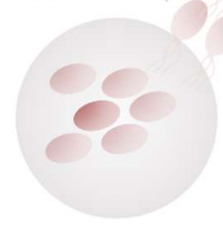

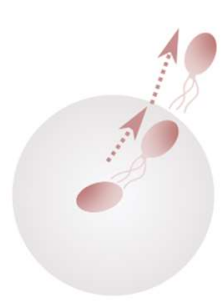

Detachment event

(B)

Residence time on particle, $\mathrm{T}$

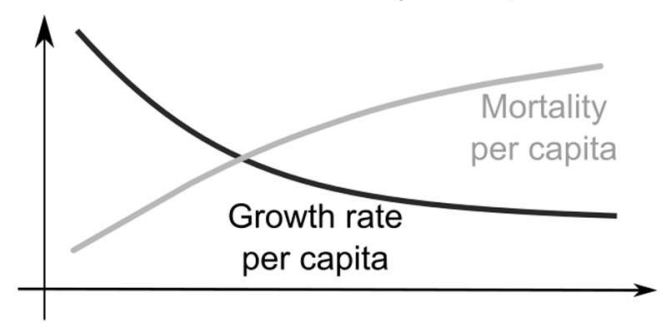

Residence time on particle, $\mathrm{T}$

(C)
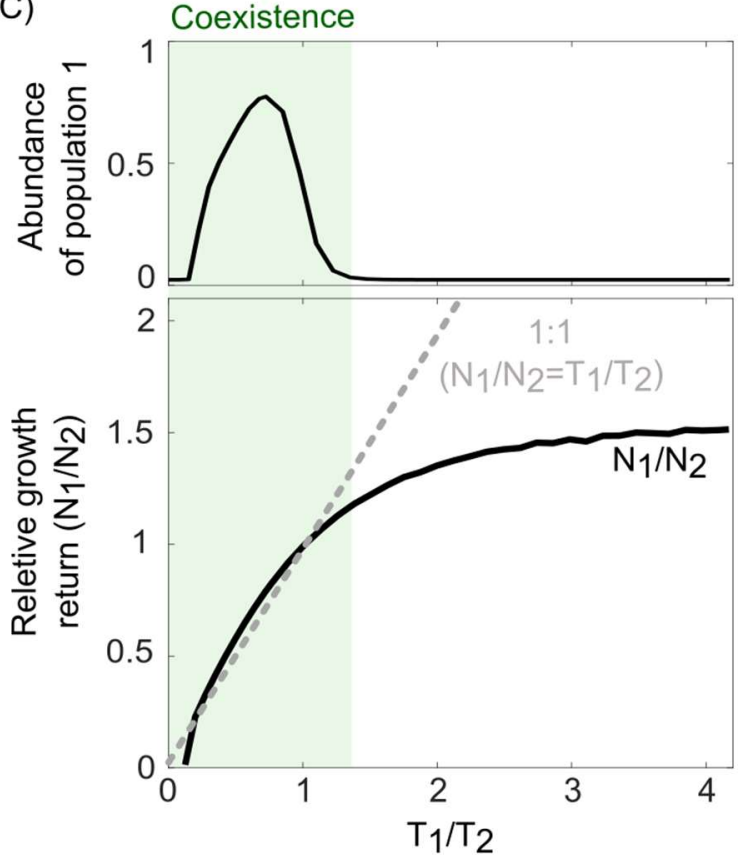

Figure 3. The trade-off between bacterial growth return and survival on the particles determine the coexistence range of competing populations. (A) The growth return of a single cell on a particle is calculated based on the number of offsprings produced during a single attachment/detachment event. (B) The growth rate on particles slows down as the particle is populated by offspring cells or new attaching cells that limit the net growth return from a particle. (C) The ratio of growth returns and survival of population one to population two per capita as a function of their radios of residence times is shown. The residence time on the particle is assumed to be the inverse of each population's detachment rate. The relative abundance of population one is shown for its corresponding simulations. The data are shown for the simulations where detachment rate of population 2 was kept constant at $0.2 \mathrm{hr}^{-1}$. Constant residence time for the population two (4hr) is considered while varying the residence time of the first population across simulations. Symbols represent the data from at the individual particle levels, while the solid black line is the average value over many particles. 1:1 line represents a coarse-grained model for the coexistence criteria of two competing populations. 


\section{Environmental fluctuations stabilize and enhance the diversity of dispersal strategies:}

286 The existence of a unique optimal strategy, even in the presence of particle-wide mortality (Figure $2872 \mathrm{~A}$ ), suggests that the coexistence that we observed between populations with different 288 detachment rates (Figure 2B) may not be evolutionarily stable. However, in the oceans, both the 289 abundance of particles and the density of predators (such as phage) exhibit temporal and spatial 290 fluctuations ${ }^{32-34}$, in turn affecting the foraging dynamics of particle-associated bacterial 291 populations. We used our model to study how the particle-wide mortality rate affects the likelihood 292 of two particle-associated bacterial populations to coexist (see Methods). Surprisingly, we found 293 that a higher mortality rate enhances the range of different detachment rates that may coexist 294 (Figure 4A). At low mortality rates, slow detaching populations outcompete faster ones, as it is 295 more advantageous to stay longer on particles and grow, i.e., these populations derive higher net 296 growth returns. However, a higher mortality rate on particles allows faster-detaching populations 297 to instead gain an advantage over the slow-detaching populations, since they can better avoid 298 particle-wide mortality events.

We extended our model to ask how variation in the total number of particles (or particle abundance) affect population dynamics and the coexistence range of populations with different dispersal strategies. The results indicated that an intermediate number of particles maximize the likelihood of coexistence of two populations with different dispersal strategies (Figure 4A). Here, we simulated a range of particle abundances, between 1 to 80 particles $\mathrm{L}^{-1}$, which corresponds to the commonly observed range of particle abundances in aquatic environments (mean 25 particles $\mathrm{L}^{-1}$; Figure S4). Low particle abundances (0 to $\left.20 \mathrm{~L}^{-1}\right)$ promote the growth of slow detaching populations while at high particle abundances, fast detaching populations dominate. The reason for this is the following: at particle abundances less than $20 \mathrm{~L}^{-1}$, the probability of freeliving cells finding and attaching to particles is less than $50 \%$ of the probability at high particle abundances (100 $\mathrm{L}^{-1}$ in Figure S5). This makes particle search times very high, thus explaining how slow detaching strategies have an advantage. As the number of particles increases, the entire system can support more cells (has a higher carrying capacity). This drives a decrease in particle search times, and thus increasingly advantages faster detaching strategies. Interestingly, our results indicate that the optimal detachment rate $\left(d_{o p t}\right)$ is affected by the particle abundance and increases with the number of particles in the system (Figure 4B). We thus

317 hypothesized that fluctuations in particle abundance may also induce fluctuations in the optimal 318 detachment rate, such that no specific detachment rate would be uniquely favored at all times. 
319 Such a "fluctuating optimum" may contribute to higher bacterial diversity on marine particles. To

320 test this hypothesis using our model, we simulated competition between 100 populations with

321 different detachment rates under a periodically varying particle abundance (Figure 4C). The

322 chosen frequencies of variation in particle abundance $\left(F_{\mathrm{p}}\right)$ were selected to be consistent with the

323 observed frequencies in the ocean, ranging between 10 to $100 \mathrm{hr}$ (Figure S6) ${ }^{35}$. We quantified

324 the range of detachment rates, a proxy for bacterial diversity, that could coexist at equilibrium

325 (Figure 4D). The results revealed that the scenario with fastest fluctuations in particle numbers

$326 \quad\left(F_{p}=10 \mathrm{hr}^{-1}\right)$ supported higher diversity among populations with different detachment rates (Figure

327 4D). Consistent with the fluctuation periods observed in the ocean, our simulations showed that

328 fluctuation at the daily scale is sufficient to support the coexistence of different dispersal

329 strategies. Overall, our model provides a framework to study how environmental fluctuations

330 contribute to observed diversity in the dispersal strategies of particle-associated populations in

331 marine environments. 


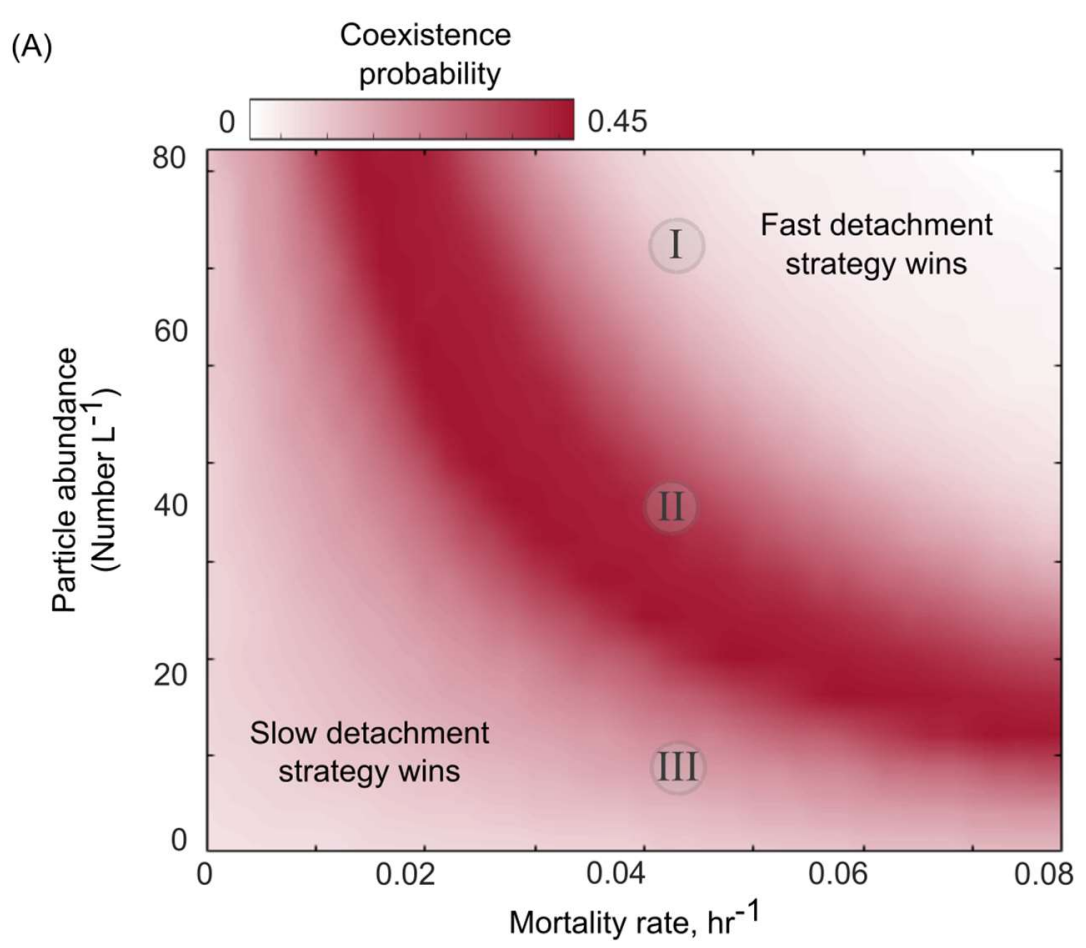

(C)

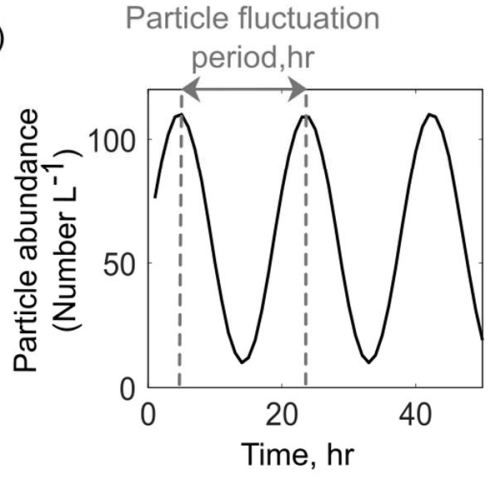

(D)

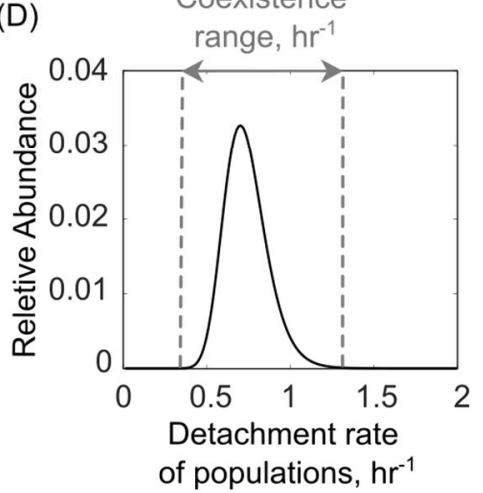

(B) Abundance

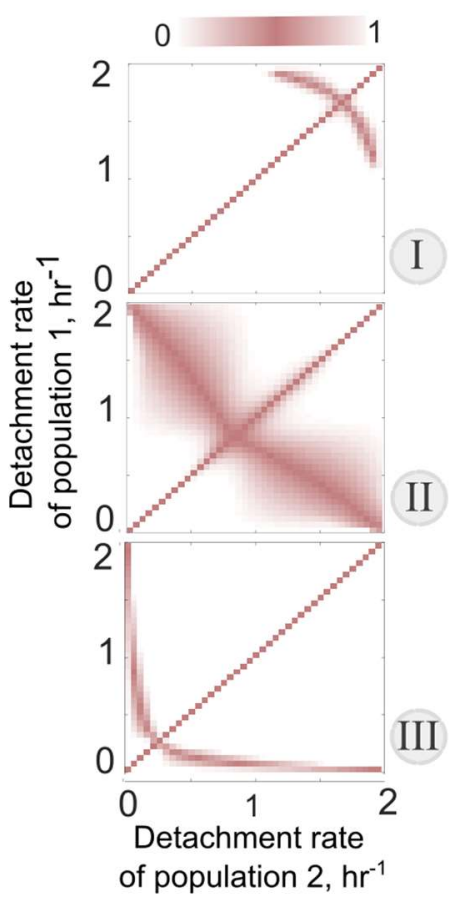

(E)

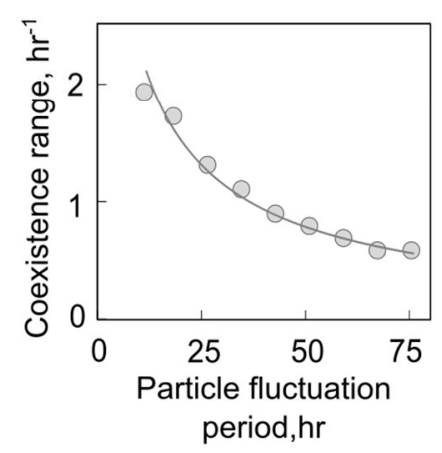

Figure 4. Particle abundance and predation rate shape the coexistence of populations

335 with different dispersal strategies on the particle system. (A) The coexistence probability is

336 shown for a range of particle abundances and predation rates. The coexistence probability is

337 calculated by performing multiple competition experiments across populations with various

338 detachment rates and quantifying the number of conditions that the coexistence between two

339 populations is found. (B) For three particle abundances in Figure 4A, the relative abundance of

340 population one is shown in competition experiments of two populations. The numbers in circles

341 refer to conditions in Figure 4A. Simulations are assumed to be at the equilibrium when no

342 changes in the size of either population are observed. The area with white color represents the

343 conditions where either one of the populations is extinct. (C) A sine function is introduced to 
represent particle abundance fluctuations. (D) The coexistence range represents a range of detachment rates for populations that coexist at the equilibrium. Populations with relative abundances less than $5 \%$ of the most abundant population is assumed extinct. (E) The coexistence range is shown as a function of particle fluctuation period. The attachment rate and mortality rates are assumed to be $\sim 0.0005 \mathrm{hr}^{-1}$ and $\sim 0.045 \mathrm{hr}^{-1}$.

\section{Discussion}

In this study, we have shown a mechanism by which diverse dispersal strategies can coexist among bacterial populations that colonize and degrade particulate organic matter (POM) in marine environments using a mathematical model. In our model, coexistence among populations with different dispersal strategies emerges from a trade-off between growth return and the probability of survival on particles. Such a trade-off determines the net number of detaching cells from particles that disperse into the bulk environment and colonize new particles. While slow detaching populations are able to increase their growth return on particles and produce a relatively high number of offspring, they also experience higher mortality on particles that reduces their ability to colonize new particles. In contrast, faster-detaching populations can better avoid mortality by spending less time on particles, but this comes at the expense of lowering their growth return on a particle. Such populations can instead disperse and colonize a larger fraction of fresh yet-unoccupied particles. Interestingly, our results indicated that in the absence of mortality on particles, no coexistence is expected and there is a single dispersal strategy that provides the highest fitness advantage over dispersing populations, indicating that mortality on a particle is a key factor for the emergence of diverse dispersal strategies. Such correlated mortality with dispersal is the direct result of spatial structures created by particle-associated lifestyle, unlike the planktonic phase where predation probability per capita is expected to be uniform among planktonic cells. This study expands on the existing knowledge that spatial structure plays a critical role in promoting bacterial diversity in nature ${ }^{36-38}$, by incorporating the idea of particle-wide predation, which are events of correlated predation of an entire population on a particle. Such correlated predation could be an ecologically relevant mechanism that explains, in part, why we

373 observe a higher diversity in particle-associated bacteria than planktonic bacteria in nature ${ }^{10-12}$.

374 Our model assumes a general form of predation on particles that is insensitive to population type.

375 However in the context of viral infection, field observations often show high strain specificity ${ }^{39-42}$ 376 that is likely to contribute to higher diversity in particle-associated populations. Viral infection act 
as a driving force to create a continuous succession of bacterial populations on particles by replacing phage exposed populations with less susceptible ones.

Consistent with the literature on optimal foraging theory ${ }^{14,43,44}$, our model predicts the existence of an optimal foraging strategy for bacterial population colonizing particles in marine environments. Building on previous studies ${ }^{26}$ that show the optimal detachment rate is a function of search time for new resources, our study suggests that optimal detachment rate could be significantly impacted by the predation rate on particles. Our results indicated that a high mortality rate on particles shifts the optimal foraging strategy to populations with fast detachment rates. Interestingly, we showed that the variability in optimal detachment rate due to environmental fluctuations in particle number and predation rate, could lead to evolutionarily stable coexistence among diverse dispersal strategies. Our results indicate that in the absence of any environmental fluctuations, there is a unique optimal dispersal strategy. However, the optimal dispersal strategy depends on the abundance of particles, and thus fluctuations in their abundance at ecological timescales could sustain multiple dispersal strategies for long times. This finding is consistent with previous theoretical and empirical studies showing that environmental fluctuations such as light and temperature may lead to the stable coexistence of species ${ }^{45-48}$. Our model also predicts a loss of diversity when particle abundances significantly increase, consistent with field observations from algal blooms ${ }^{49-51}$.

While we simplified bacterial colonization dynamics on particles by only considering competitive growth kinetics, variants of our model suggest that coexistence between different dispersal strategies is also expected under more complex microbial interactions that are observed on marine particles, including cooperative growth dynamics (Figure S7). Overall, our model provides a reliable framework to further study how diverse dispersal strategies and mortality could contribute to the emergence of complex community dynamics on marine particles and how environmental factors impact microbial processes in regulating POM turnover at the ecosystem

404 level. 


\section{Methods}

412 In this study, a population-based model is developed that represents the interactions between the 413 bacterial cells with different detachment rates and particles in a chemostat system, where the total 414 number of particles is kept constant. The following provides a detailed procedure of the modeling 415 steps as represented schematically in Figure 1.

\section{Modeling Population Dynamics on Particles}

418 Our model simulates the dynamics of two competing particle-associated populations $\left(B_{p}\right)$ that 419 colonize the same set of particles. Two populations ( $i$ and $j$ ) are assumed to be identical, except 420 for their detachment rates, $d$, from a particle $\left(d_{i} \neq d_{j}\right)$. The variations in particle-associated 421 populations are determined by the rate at which cells attach to particles $(\alpha)$ from the free-living 422 population $\left(B_{F}\right)$, the growth rate of attached cells $(\mu)$, detachment rate $(d)$, and the mortality rate 423 on particles $\left(m_{p}\right)$ :

$$
\frac{\partial B_{p, n, i}}{\partial t}=\alpha_{i} B_{F i}+\mu_{i} B_{p, n, i}-d_{i} B_{p, n, i}-m_{p, i} B_{p, n, i}
$$

where $n$ represents the particle index and its associated population, $i$. Equation 3 can be formulated for any other population at the same particle. In a system with $N_{p}$ particles and $\mathrm{M}$ populations, we numerically solve a finite set of equations $\left(N_{p} \times \mathrm{M}\right)$ at each time interval.

428 From the mass conservation principle, the free-living bacterial population can also be calculated 429 from the net rate of cells that detach from the particles and the total number of cells that attach to 430 the particles. The model also considers a mortality rate for the free-living cells due to starvation $431\left(m_{F}\right)$ :

$$
\frac{\partial B_{F i}}{\partial t}=\sum_{n=1}^{N_{p}} d_{i} B_{p, n, i}-N_{p} \alpha_{i} B_{F i}-m_{F i} B_{F i}
$$

433 The simulations are run over time until an equilibrium is reached and no noticeable changes in

434 the size of particle-associated and free-living cells are observed $\left(\frac{\partial B_{p}}{\partial t} \sim 0 \& \frac{\partial B_{F}}{\partial t} \sim 0\right)$. 


\section{Bacteria-particle encounter rate}

437 We assume that a bacterial cell can attach to the particle it encounters and stay for a period of 438 time ("residence time"). The encounter probability of a spherical cell with radius $r_{c}$ and a spherical 439 particle with a radius of $r_{p}$ at a given time $t$ can be calculated using the hitting probability from 440 random walk theory:

$$
P_{e}(i)=\frac{R}{d} \operatorname{erfc}\left(\frac{D_{c, p}-R}{\sqrt{4 D . t}}\right)
$$

442 where $R$ is the total radius $\left(R=r_{p}+r_{c}\right)$ and $D$ is an effective diffusion coefficient $\left(D=D_{c}+D_{p}\right)$

443 for a bacterial cell $(c)$ starting at a distance $\left(D_{c, p}\right)$. The diffusion coefficient can be calculated from 444 an empirical model: $D=k_{B} T / 6 \pi \mu r$, where $k_{B} \approx 1.38 \times 10^{-23} \mathrm{JK}^{-1}$ is Boltzmann's constant, $445 \mathrm{~T}=293 \mathrm{~K}$ is ambient temperature, $\mu=1.003 \mathrm{mPas}$ is the viscosity of water at the given ambient 446 temperature. In aquatic environments, the size of marine snow $(>100 \mu m)$ is often a lot larger than

447 the cell size, we thus assume that the effective diffusion is generally controlled by cell diffusion 448 coefficient $\left(D \approx D_{c}\right)$. From Eq. 5 , we calculated the total number attaching cells to a particle at a 449 given time $(t)$ from free living cells of population $i$ by multiplying the hitting probability to the total 450 number of free-living cells.

\section{Growth and reproduction on particles}

452 In this model, we assume that the bacterial growth on the particle is competitive in which the 453 growth rate, $\mu_{i}$ is not constant but changes as a function of the number of cells on a particle. The 454 negative density-dependent growth is modeled by assuming a linear function with the total 455 number of cells on the particle $\left(N_{c}=\sum N_{c, i}\right)$,

$$
\left\{\begin{array}{c}
\mu_{i}=\mu_{\max }\left(1-\frac{N_{c}}{N_{t}}\right) \\
\mu_{i}=0, N_{c}>N_{t}
\end{array}\right.
$$

458 with $\mu_{\max }$ that indicates the growth rate of the first cell encountering the particle and $N_{t}$ that 459 represents the carrying capacity. The growth rate is assumed to be zero if more cells are 460 colonizing the particle compared to its carrying capacity. The model assumes that there is no 461 growth for free living cells. We performed a sensitivity analysis to competitive growth kinetic parameterizations (maximum growth rate $\mu_{\max }$ and carrying capacity, $\mathrm{N}_{\mathrm{t}}$ ) and showed that the 
coexistence among bacterial detachment strategies is robust for a wide range of parameters (Figure S8).

The biomass accumulation at the particle for population $i$ is assumed to be proportional to the cells dry mass, $B_{c}$ and therefore the net growth in the cell biomass can be described as $\frac{d B_{c}}{d t}=$

$467 B_{c} \mu_{i}$. In order for particle associated cells to reproduce offspring, the total biomass accumulation 468 at the population level should exceed the dry mass of a single cell $\left(d_{m}\right)$. The total number of 469 offspring $\left(B_{i}\right)$ on a particle can then be calculated as:

$$
B_{i}=\frac{B_{c} \mu_{i}}{d_{m}}
$$

471 The model assumes that the offspring reproduction only occurs when the total accumulation is 472 larger than the dry mass of a single cell $\left(B_{c} \mu_{i}>d_{m}\right)$, otherwise, the biomass is accumulated over 473 time until the criteria are met for reproduction.

\section{Bacterial mortality}

475 In the model, a general form of mortality on particles is considered that accounts for mortality 476 induced by predation or particle sinking and becoming inaccessible at deeper layers of the water 477 column in the ocean. A constant fraction of particles that represent the mortality rate $\left(m_{p}\right)$ is 478 uniform randomly selected at each time step and their associated cells are removed from the 479 particle. Then, the uncolonized particle is again introduced into the system and colonized by free480 living populations $\left(B_{p, n, i}=0\right)$.

481 Mortality of free-living cells are assumed to be caused by loss of biomass over a prolonged period 482 of starvations from the absence of substrate uptake in the free-living phase. As described in Eq. 4834 , free-living cells $\left(B_{F}\right)$ lose a constant fraction of their biomass $\left(m_{F i}\right)$ every time step as the cell 484 maintenance.

\section{Particle degradation and turnover}

486 We assume that a particle contains a finite amount of resources that is degraded by bacterial cells 487 with a constant yield of converting the resources into biomass. From a previous study, we assume 488 that the yield is about $5 \%$ and a significant fraction of particle degradation products are lost to the 489 environment before being taken up by the cells ${ }^{17}$. 


\section{Optimal residence time from optimal foraging theory}

492 Optimal foraging theory describes the dispersal behavior of microbial populations in patchy 493 environments assuming maximized growth return using the marginal value theorem. According

494 to optimal foraging theory, the growth return of particle-associated bacteria is maximized if a 495 bacterial cell detaches from the particle when its time-averaged uptake rate reaches its 496 instantaneous uptake rate. We applied this assumption to obtain the optimal residence time on 497 particles by tracking individual cells in our model and calculating their instantaneous uptake rate $498(u(t))$ on a particle from the attachment time $\left(t_{a}\right)$ to detachment. The residence time $\left(t_{r}\right)$ is 499 considered optimal when the following equation is satisfied:

500

$$
u\left(t_{r}\right)=\sum_{t_{a}}^{t_{r}} \frac{u\left(t_{r}\right)}{\left(\tau_{s}+\left(t_{r}\left(\tau_{s}\right)-t_{a}\right)\right)}
$$

503 where $\tau_{s}$ is the search time and a function of the number of particles in the system. We calculated 504 the search time from Eq.5 when the probability of the cell and particle encounter is above $95 \%$ 505 (Figure S5). 


\section{References}

1. Azam, F. \& Malfatti, F. Microbial structuring of marine ecosystems. Nat. Rev. Microbiol. 5, 782-791 (2007).

512 2. Young, I. M. \& Crawford, J. W. Interactions and self-organization in the soil-microbe complex. Science 304, 1634-1637 (2004).

3. Ghilarov, A. M. The Paradox of the Plankton Reconsidered; Or, Why Do Species Coexist?

4. Shoresh, N., Hegreness, M. \& Kishony, R. Evolution exacerbates the paradox of the plankton. Proc. Natl. Acad. Sci. U. S. A. (2008). doi:10.1073/pnas.0803032105

5. Hutchinson, G. E. The Paradox of the Plankton. Am. Nat. (1961). doi:10.1086/282171

6. Goyal, A. \& Maslov, S. Diversity, Stability, and Reproducibility in Stochastically Assembled

7. Rodriguez-Valera, F. et al. Explaining microbial population genomics through phage predation. Nat. Rev. Microbiol. (2009). doi:10.1038/nrmicro2235

8. Muscarella, M. E., Boot, C. M., Broeckling, C. D. \& Lennon, J. T. Resource heterogeneity structures aquatic bacterial communities. ISME J. (2019). doi:10.1038/s41396-019-0427-

9. Saleem, M., Fetzer, I., Harms, H. \& Chatzinotas, A. Diversity of protists and bacteria determines predation performance and stability. ISME J. (2013). doi:10.1038/ismej.2013.95

10. Milici, M. et al. Diversity and community composition of particle-associated and free-living bacteria in mesopelagic and bathypelagic Southern Ocean water masses: Evidence of dispersal limitation in the Bransfield Strait. Limnol. Oceanogr. (2017). doi:10.1002/Ino.10487

11. Ganesh, S., Parris, D. J., Delong, E. F. \& Stewart, F. J. Metagenomic analysis of sizefractionated picoplankton in a marine oxygen minimum zone. ISME J. (2014). doi:10.1038/ismej.2013.144

12. Crespo, B. G., Pommier, T., Fernández-Gómez, B. \& Pedrós-Alió, C. Taxonomic composition of the particle-attached and free-living bacterial assemblages in the Northwest Mediterranean Sea analyzed by pyrosequencing of the 16S rRNA. Microbiologyopen (2013). doi:10.1002/mbo3.92

13. Yawata, Y., Carrara, F., Menolascina, F. \& Stocker, R. Constrained optimal foraging by 
marine bacterioplankton on particulate organic matter. Proc. Natl. Acad. Sci. (2020). doi:10.1073/pnas.2012443117

14. Fernandez, V. I., Yawata, Y. \& Stocker, R. A Foraging Mandala for Aquatic Microorganisms. ISME Journal (2019). doi:10.1038/s41396-018-0309-4

15. Grossart, H. P., Kiørboe, T., Tang, K. \& Ploug, H. Bacterial colonization of particles: Growth and interactions. Appl. Environ. Microbiol. (2003). doi:10.1128/AEM.69.6.3500-3509.2003

16. Yawata, Y. et al. Competition-dispersal tradeoff ecologically differentiates recently

17. Ebrahimi, A., Schwartzman, J. \& Cordero, O. X. Cooperation and spatial self-organization determine rate and efficiency of particulate organic matter degradation in marine bacteria. Proc. Natl. Acad. Sci. U. S. A. (2019). doi:10.1073/pnas.1908512116

18. Boeuf, D. et al. Biological composition and microbial dynamics of sinking particulate organic matter at abyssal depths in the oligotrophic open ocean. Proc. Natl. Acad. Sci. U. S. A. (2019). doi:10.1073/pnas.1903080116

19. Dupont, C. L. et al. Genomes and gene expression across light and productivity gradients in eastern subtropical Pacific microbial communities. ISME J. (2015). doi:10.1038/ismej.2014.198

21. Proctor, L. M. \& Fuhrman, J. A. Roles of viral infection in organic particle flux. Mar. Ecol. Prog. Ser. (1991). doi:10.3354/meps069133

22. Weinbauer, M. G. et al. Viral ecology of organic and inorganic particles in aquatic systems: Avenues for further research. Aquat. Microb. Ecol. (2009). doi:10.3354/ame01363

23. Leventhal, G. E., Ackermann, M. \& Schiessl, K. T. Why microbes secrete molecules to modify their environment: The case of iron-chelating siderophores. J. R. Soc. Interface (2019). doi:10.1098/rsif.2018.0674

24. Frazier, Z. \& Alber, F. A computational approach to increase time scales in brownian

572 25. Yawata, Y. et al. Competition-dispersal tradeoff ecologically differentiates recently dynamics-based reaction-diffusion modeling. J. Comput. Biol. (2012). doi:10.1089/cmb.2012.0027 speciated marine bacterioplankton populations. Proc. Natl. Acad. Sci. 111, 5622-5627 (2014). 
575 26. Yawata, Y., Carrara, F., Menolascina, F. \& Stocker, R. Constrained optimal foraging by marine bacterioplankton on particulate organic matter. Proc. Natl. Acad. Sci. U. S. A. (2020). doi:10.1073/pnas.2012443117

27. Charnov, E. L. Optimal foraging, the marginal value theorem. Theor. Popul. Biol. (1976). doi:10.1016/0040-5809(76)90040-X

28. Levin, S. A. \& Paine, R. T. Disturbance, patch formation, and community structure. Proc. Natl. Acad. Sci. U. S. A. (1974). doi:10.1073/pnas.71.7.2744

29. Kneitel, J. M. \& Chase, J. M. Trade-offs in community ecology: Linking spatial scales and species coexistence. Ecology Letters (2004). doi:10.1046/j.1461-0248.2003.00551.x

30. Tilman, D. Competition and biodiversity in spatially structured habitats. Ecology (1994). doi:10.2307/1939377

31. Amarasekare, P. Competitive coexistence in spatially structured environments: A synthesis. Ecology Letters (2003). doi:10.1046/j.1461-0248.2003.00530.x

32. Nilsson, E. et al. Genomic and Seasonal Variations among Aquatic Phages Infecting the

33. Garin-Fernandez, A., Pereira-Flores, E., Glöckner, F. O. \& Wichels, A. The North Sea goes viral: Occurrence and distribution of North Sea bacteriophages. Mar. Genomics 41, 31-41 (2018).

34. Luo, E., Aylward, F. O., Mende, D. R. \& Delong, E. F. Bacteriophage distributions and temporal variability in the ocean's interior. MBio 8, (2017).

35. Lampitt, R. S., Hillier, W. R. \& Challenor, P. G. Seasonal and diel variation in the open ocean concentration of marine snow aggregates. Nature (1993). doi:10.1038/362737a0

36. Eckburg, P. B. et al. Microbiology: Diversity of the human intestinal microbial flora. Science (80-. ). (2005). doi:10.1126/science.1110591

37. Zhang, Z. et al. Spatial heterogeneity and co-occurrence patterns of human mucosalassociated intestinal microbiota. ISME J. (2014). doi:10.1038/ismej.2013.185

38. Young, I. M. \& Crawford, J. W. Interactions and self-organization in the soil-microbe complex. Science (2004). doi:10.1126/science.1097394

39. Holmfeldt, K., Middelboe, M., Nybroe, O. \& Riemann, L. Large variabilities in host strain susceptibility and phage host range govern interactions between lytic marine phages and their Flavobacterium hosts. Appl. Environ. Microbiol. (2007). doi:10.1128/AEM.01399-07

40. Roux, S. et al. Assessing the diversity and specificity of two freshwater viral communities through metagenomics. PLoS One (2012). doi:10.1371/journal.pone.0033641 
41. Suttle, C. A. \& Chan, A. M. Dynamics and distribution of cyanophages and their effect on marine Synechococcus spp. Appl. Environ. Microbiol. (1994). doi:10.1128/aem.60.9.31673174.1994

42. Suttle, C. A. Viruses in the sea. Nature (2005). doi:10.1038/nature04160

43. Taylor, J. R. \& Stocker, R. Trade-offs of chemotactic foraging in turbulent water. Science (80-. ). 338, 675-679 (2012).

44. Vetter, Y. A., Deming, J. W., Jumars, P. A. \& Krieger-Brockett, B. B. A predictive model of bacterial foraging by means of freely released extracellular enzymes. Microb. Ecol. (1998). doi:10.1007/s002489900095

45. Litchman, E. Competition and coexistence of phytoplankton under fluctuating light: Experiments with two cyanobacteria. Aquat. Microb. Ecol. (2003). doi:10.3354/ame031241

46. Li, L. \& Chesson, P. The effects of dynamical rates on species coexistence in a variable environment: The paradox of the plankton revisited. Am. Nat. (2016). doi:10.1086/687111

47. Catorci, A., Piermarteri, K., Penksza, K., Házi, J. \& Tardella, F. M. Filtering effect of

48. Sousa, W. P. Disturbance in Marine Intertidal Boulder Fields: The Nonequilibrium Maintenance of Species Diversity. Ecology (1979). doi:10.2307/1936969

49. Teeling, H. et al. Substrate-controlled succession of marine bacterioplankton populations induced by a phytoplankton bloom. Science (80-. ). (2012). doi:10.1126/science.1218344

50. West, N. J., Obernosterer, I., Zemb, O. \& Lebaron, P. Major differences of bacterial diversity and activity inside and outside of a natural iron-fertilized phytoplankton bloom in the Southern Ocean. Environ. Microbiol. (2008). doi:10.1111/j.1462-2920.2007.01497.x community in the southern North Sea as revealed by metatranscriptomic approaches. 


\section{Supplementary Figures}

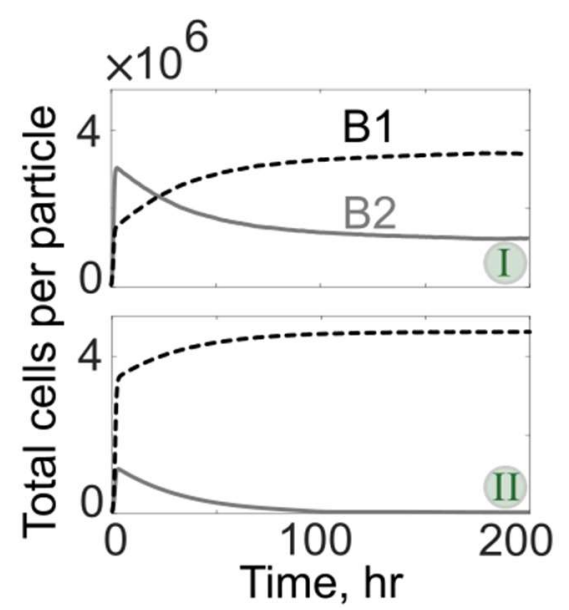

642 Figure S1. Two examples of population dynamics are shown wherein both populations reach a

643 stable coexistence (I), while in the other scenario (II), one population is extinct. The detachment

644 rates of the populations B1 and B2 in scenarios I and II are $\left(0.7 \mathrm{hr}^{-1}, 0.2 \mathrm{hr}^{-1}\right)$ and $\left(0.7 \mathrm{hr}^{-1}, 1 \mathrm{hr}^{-1}\right)$,

645 respectively. These population dynamics correspond to the conditions simulated in Figure $2 \mathrm{~A}$. 


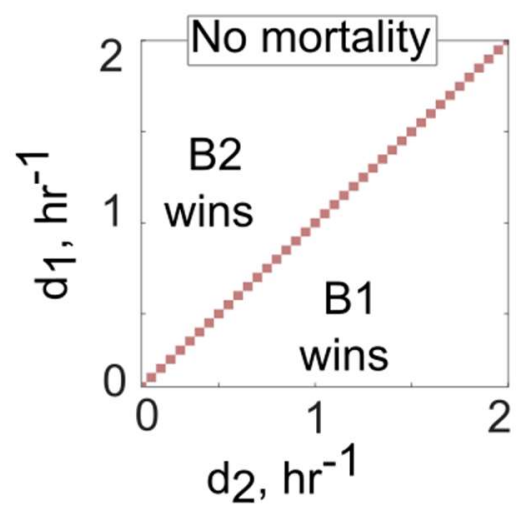

656 Figure S2. The relative abundance of population one is shown when no mortality on particles is 657 considered for competition experiments of two populations with different detachment rates. The 658 parameters for the simulations are selected similar to Figure 2A, except with no particle-wide 659 mortality. 


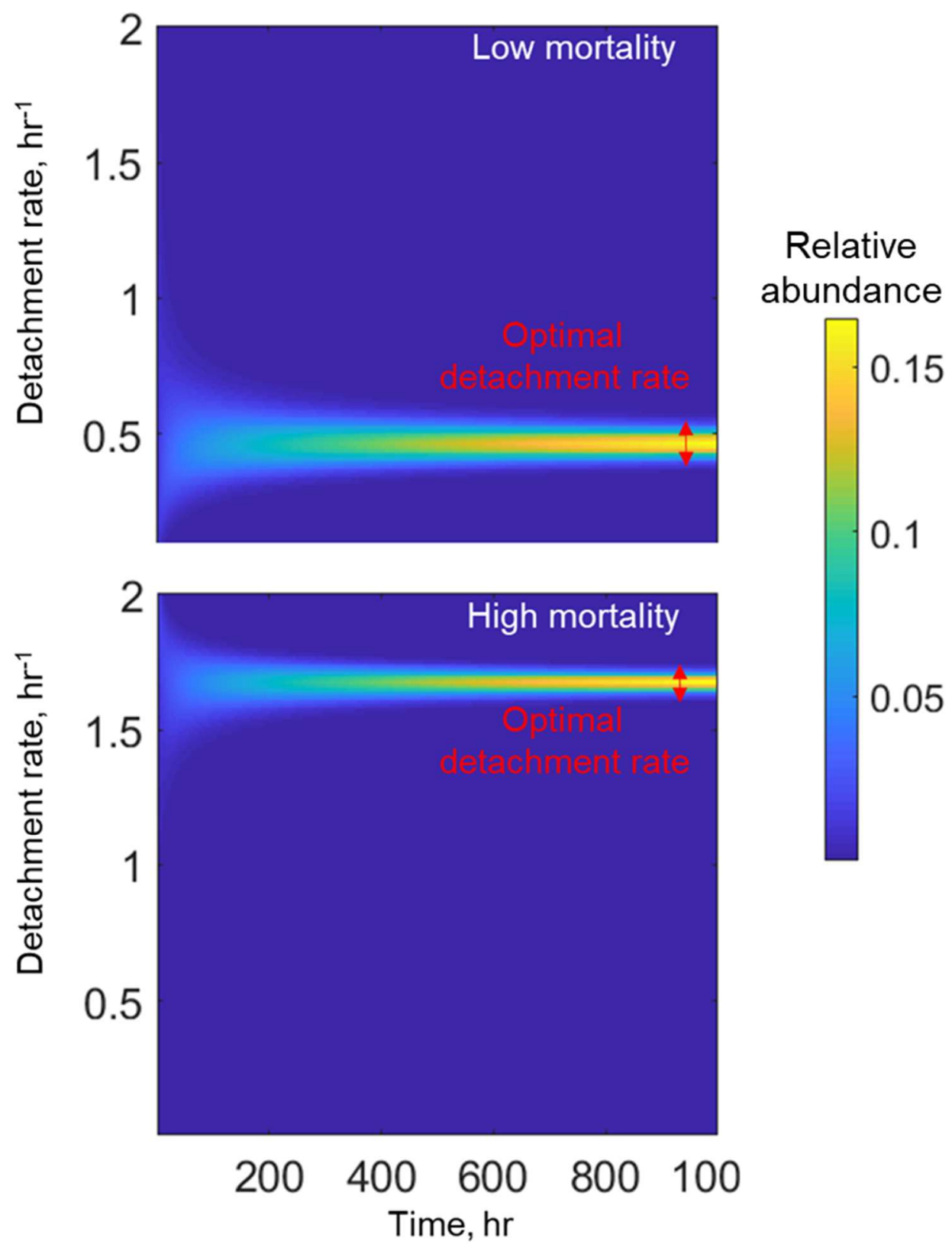

665 Figure S3. In the absence of environmental fluctuations, competition experiment between 666 populations with different detachment rates shows an emergence of an optimal detachment

667 strategy that outcompete other populations. The relative abundances of populations with

668 different detachment rates are shown over time. The simulations start with 100 populations with

669 the same relative abundances that they only differ in their detachment rates. In this simulation,

670 bacterial cells colonize 1000 particles with a constant attachment rate $\left(\sim 0.0005 \mathrm{hr}^{-1}\right)$. Two

671 mortality rates are simulated (low: $0.04 \mathrm{hr}^{-1}$ and high $0.08 \mathrm{hr}^{-1}$ ) 


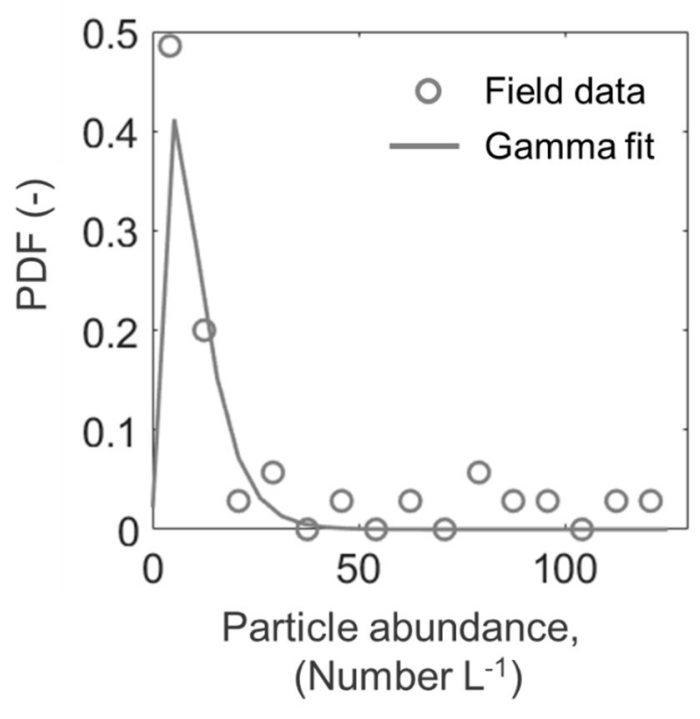

674 Figure S4. Particle abundance distributions extracted from the field observations. The particle 675 abundances are extracted over many field observations across many aquatic environments at 676 different geographical locations (Möller et al. 2012; Ashijan et al., 2005; Gallager et al., 2004;

677 Norrbin et al., 1996). The mean particle abundance over these field data is approximately 25 per 678 liter. 
(A)

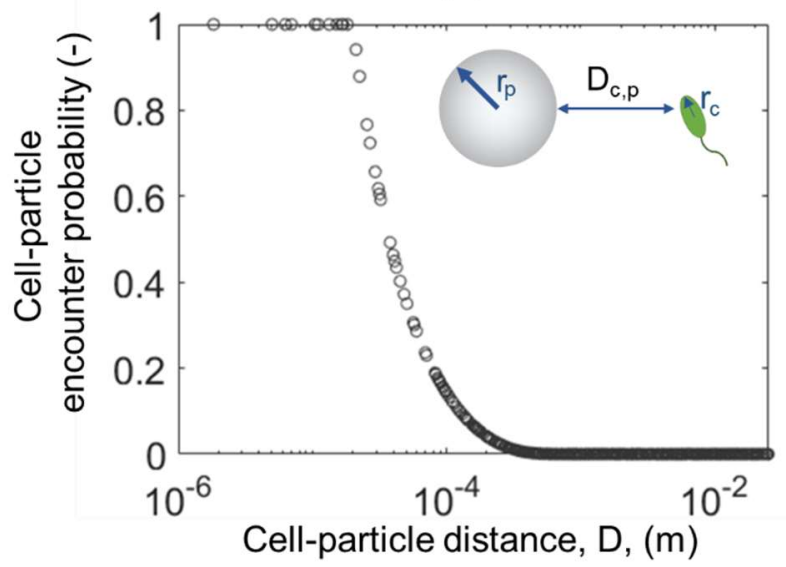

(B)

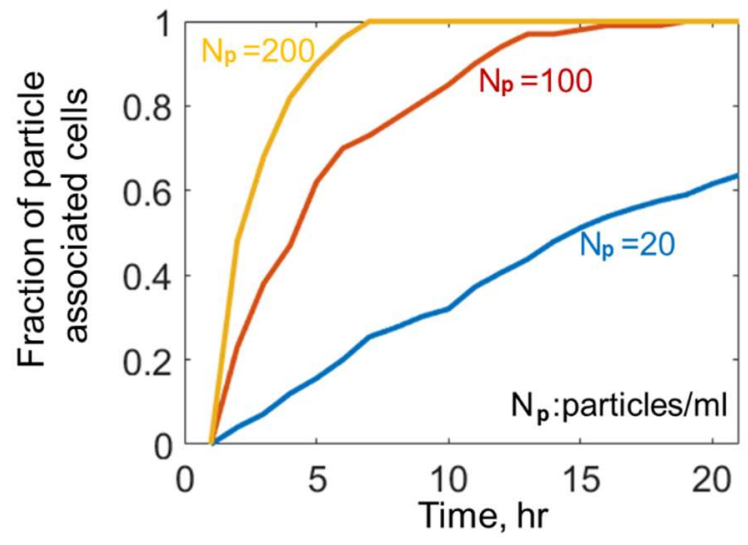

689 Figure S5. (A) Encounter probability of a bacterial cell and a particle as a function of cell-particle

690 distance, $D_{c, p}$. An analytical model based on the hitting probability of two objects from random

691 walk theory is used to obtain the encounter probability (Eq. 5). (B) The rate of free living

692 bacterial cells attachment to particles as a function of time for various number particle

693 availability in the environment. Bacterial cell and particle sizes are assumed to be 1 and $100 \mu \mathrm{m}$,

694 respectively.

695

696

697

698

699

700

701

702

703

704 
(A)

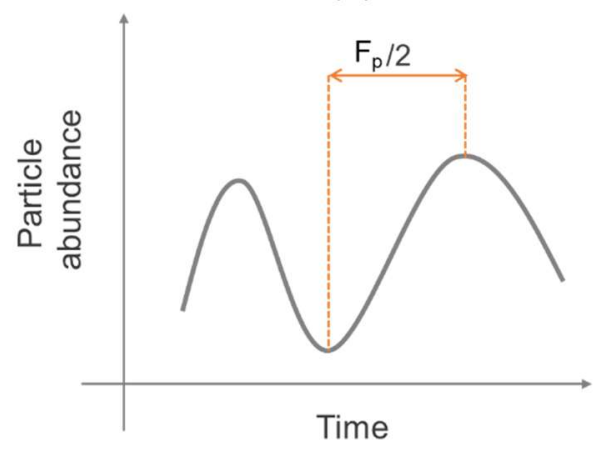

(B)

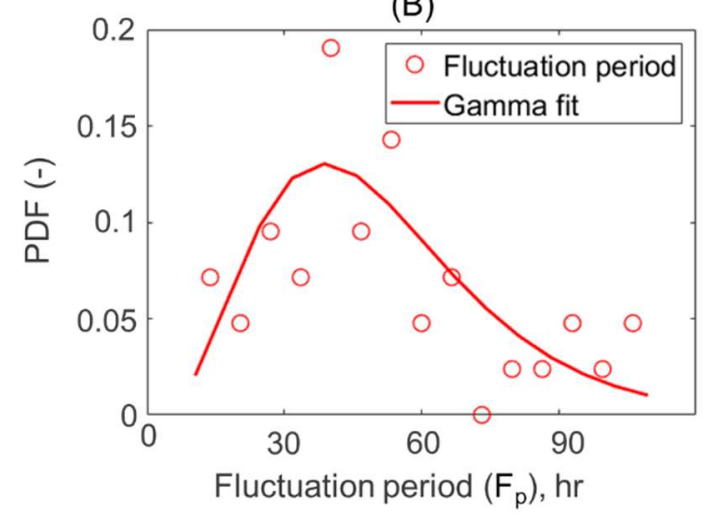

706

707 Figure S6. The durations of environmental fluctuation periods for particle abundances are

708 extracted from field ${ }^{35}$. (A) The fluctuations in particle abundance are characterized by

709 quantifying fluctuation periods from the time difference between two neighboring local minimum

710 and maximum, as illustrated in the schematic. (B) Probability distribution functions for fluctuation

711 periods in particle abundances obtained from field observations.

712

713

714

715

716

717

718 
(A)

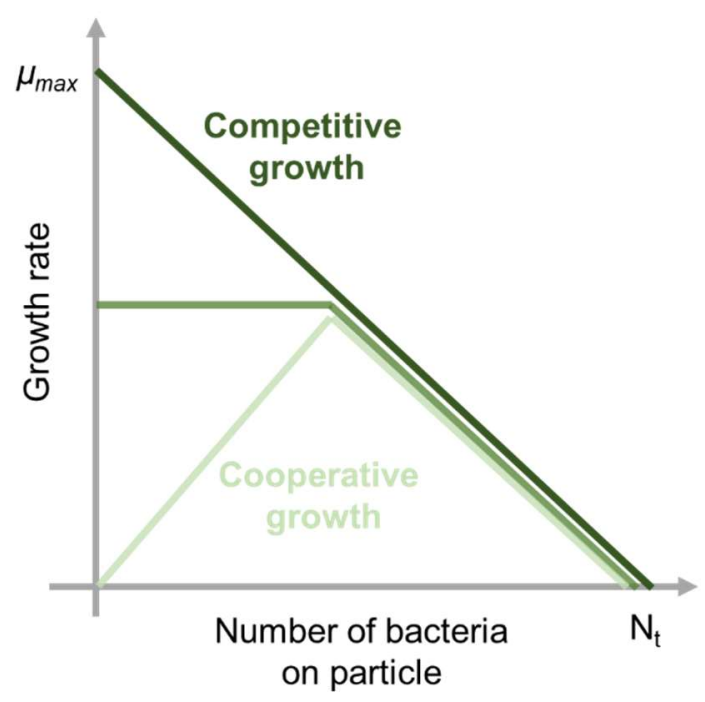

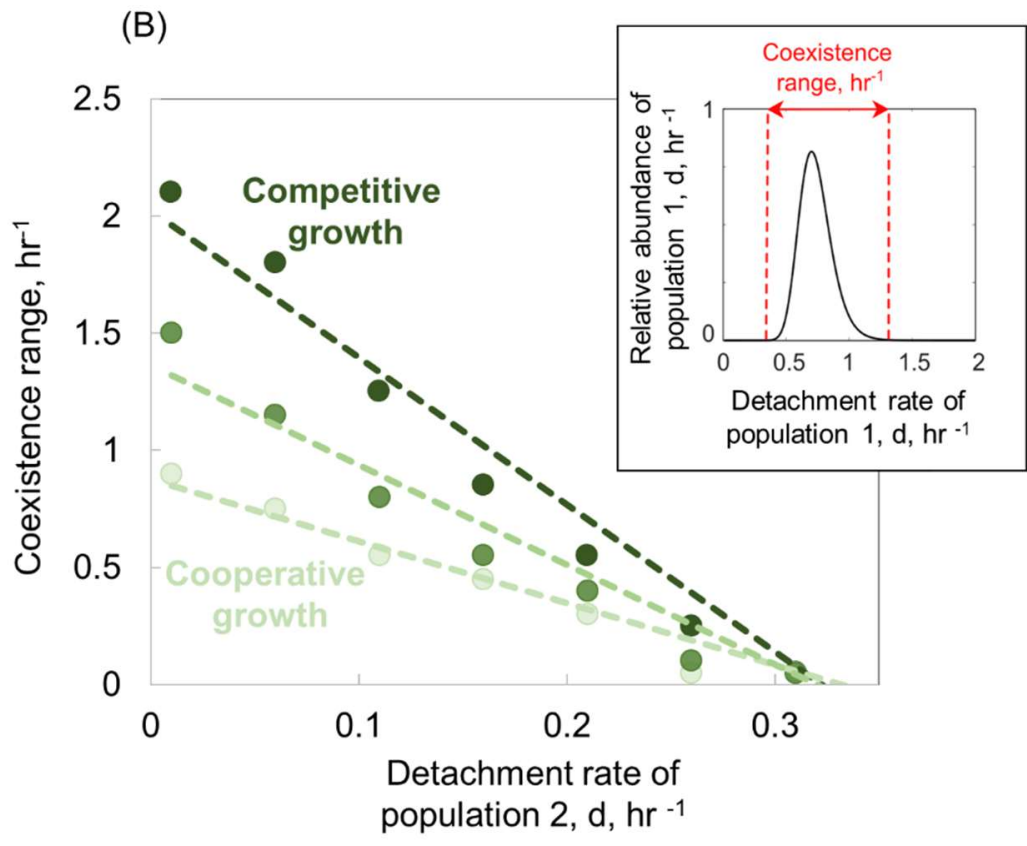

721 Figure S7. Cooperative growth kinetics restricts the coexistence range among two populations

722 with different dispersal strategies. (A) Schematic representation of various growth kinetics on

723 particles as a function of the number of bacteria on particles. (B) The coexistence range among

724 two populations is shown as a function of the detachment rate of the second population. The

725 coexistence range represents a range of detachment rates for both populations that coexist at

726 the equilibrium. Inset panel represents the relative abundance of population 1 for different

727 detachment rates. Detachment rates with relative abundances less than $5 \%$ is assumed extinct.

728 The mortally on particles is assumed $0.02 \mathrm{hr}^{-1}$. The attachment rates are kept constant at

$7290.0005 \mathrm{hr}^{-1}$. The number of particles is assumed to be $60 \mathrm{~L}^{-1}$. The carrying capacity and maximum

730 growth rates are assumed, $\mathrm{N}_{\mathrm{t}}=5 \times 10^{6}$ and $\mu_{\max }=0.50 \mathrm{hr}^{-1}$, respectively. 


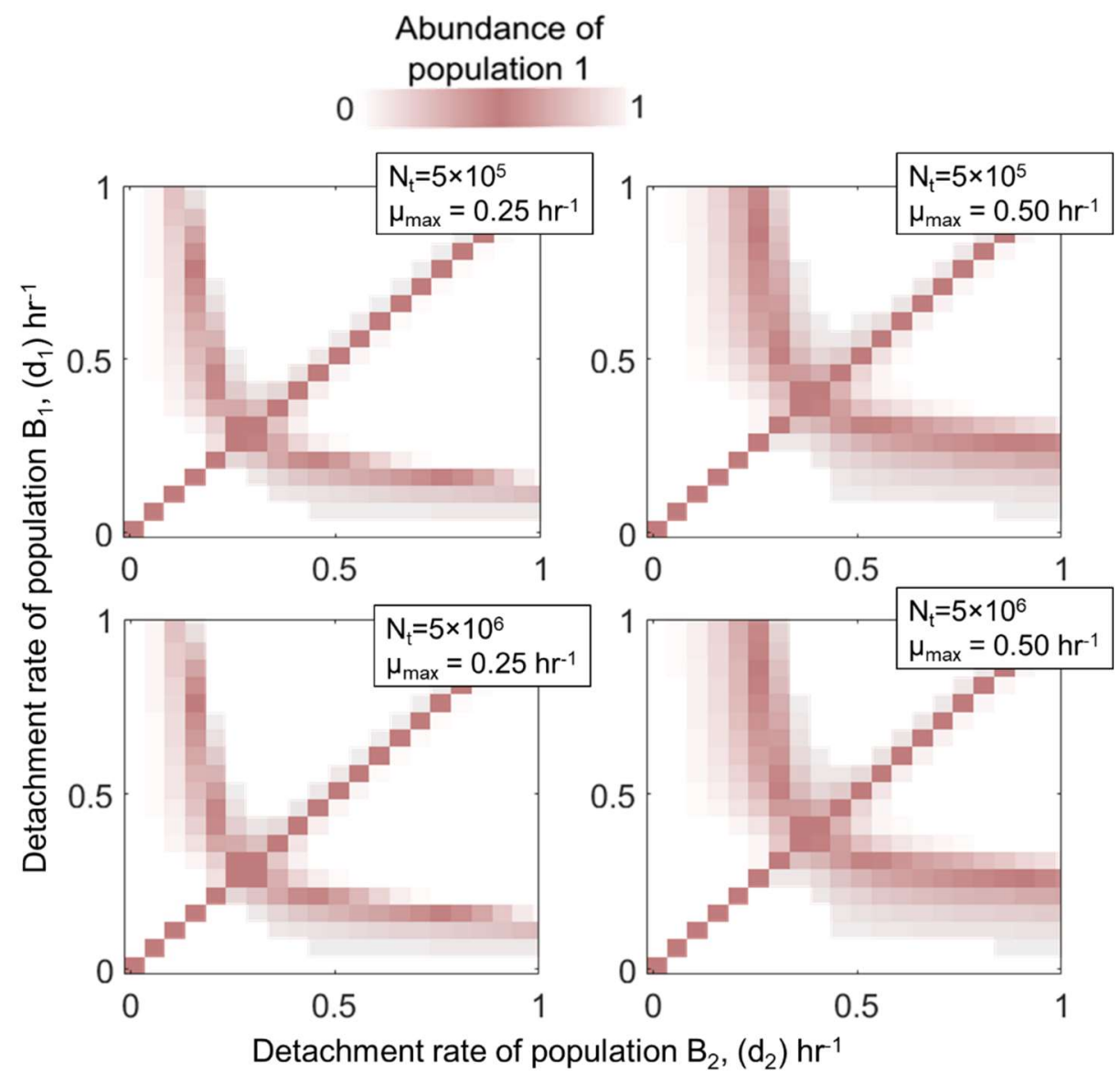

733 Figure S8. The sensitivity of coexistence among bacterial detachment strategies to competitive 734 growth kinetic parameterizations (Eq. 6: maximum growth rate $\mu_{\max }$ and carrying capacity, $\mathrm{N}_{\mathrm{t}}$ ).

735 The relative abundance of population one is shown for competition experiments of two 736 populations with different detachment rates and for two different values of maximum growth rates 737 and carrying capacities. The area with white color represents the conditions where either one of 738 the populations is extinct. The mortally on particles is assumed $0.02 \mathrm{hr}^{-1}$. The attachment rates are 739 kept constant at $0.0005 \mathrm{hr}^{-1}$. The number of particles is assumed to be $60 \mathrm{~L}^{-1}$. 